\title{
PROCEDIMENTI DI MISURAZIONE, PREDICIBILITÀ E DETERMINISMO NELLE LEGGI DELLA FISICA
}

\author{
FAUSTO BORGONOVI (*)
}

\begin{abstract}
RIASSUNTO. - I dati finanziari e la realtà fisica vengono descritti mediante modelli matematici. Mentre ciò sembra del tutto ovvio per i fenomeni naturali (alla luce dei molti e importanti progressi compiuti nella fisica dell'ultimo secolo), la questione della modellizzazione matematica dei dati economico-finanziari è ancora oggetto di dibattito. In particolare, il problema della prevedibilità di alcuni eventi viene qui discussa e si stabilisce la sua relazione con un processo presunto di misurazione oggettiva.
\end{abstract}

$* * *$

ABSTRACT. - Financial data and physical reality are described in terms of mathematical models. While this seems quite obvious for the natural phenomena (in the light of the many and important progress done in the last century physics), the question of the mathematical modelization of the former is still under debate. In particular, the problem of the predictability of some event is discussed and its relationship with a presumed objective measurement process established.

Mi è stato chiesto, in qualità di fisico di partecipare a questa giornata dedicata all'economia quantitativa con uno sguardo, in particolare, ai suoi possibili aspetti tecnologici.

Sempre come fisico, mi è stato richiesto di mettere in luce quali sono le caratteristiche che rendono questa disciplina fortemente predittiva rispetto, ad esempio, ad una disciplina catalogabile nell'ambito delle scienze umane. Mi occuperò in modo particolare non tanto degli scopi della ricerca in fisica quanto, piuttosto, del suo presunto potere predittivo e, se possibile, di comprenderne le ragioni.

(*) Dipartimento di Matematica e Fisica, Università Cattolica, Brescia. 
La fisica, come molte altre discipline naturali, è una scienza sperimentale, deve cioè essere continuamente verificata in laboratorio. Se questo fatto poteva essere fuori discussione per un fisico vissuto ai tempi di Galileo o di Newton, oggi può assumere un carattere fortemente discriminante visto che gran parte della ricerca in fisica è di carattere prevalentemente teorico. Il metodo sperimentale, così come formulato da Galileo, garantisce che i risultati di certi esperimenti, descritti in modo minuzioso e sotto particolari condizioni siano riproducibili in tutti i laboratori del mondo. Una misura sperimentale, ovvero un procedimento oggettivo di confronto con una unità di misura prefissata, risulta essere scientificamente vera se può essere riprodotta, entro un certo errore sperimentale, da tutti, sotto le medesime condizioni sperimentali.

Questo però è vero solo in parte. Ad esempio l'attuale fisica di punta, quella delle particelle elementari, richiede contributi così grandi che in pratica pochi laboratori al mondo sono in grado di riprodurre tali risultati: ciò vuol forse dire che i dati ottenuti non sono presi in considerazione dalla comunità internazionale dei fisici? Assolutamente no e anche se la cosa può suscitare alcune perplessità i risultati da loro ottenuti soddisfano tutti i requisiti per poter essere considerati scientificamente validi.

L'assenza di riproducibilità non è però dettata esclusivamente da ragioni economiche: tutta l'attività scientifica che va sotto il nome di astrofisica si basa su osservazioni relative ad eventi accaduti milioni di anni fa e assolutamente non riproducibili. Ed ovviamente anche queste attività sperimentali godono di grande prestigio scientifico internazionale.

Lasciamo per il momento da parte il discorso sulla riproducibilità per concentrarci invece sul potere predittivo, o almeno presunto tale, della fisica.

Attraverso lo studio sistematico delle leggi (o equazioni) che regolano i fenomeni naturali, la fisica è riuscita a costruire alcuni semplici modelli matematici che applicati alla pur complessa realtà sono in grado di prevedere, con grande precisione, il verificarsi di certi eventi. Sappiamo esattamente a che ora sorgerà il sole domani o tra sei mesi, o quando avverrà la prossima eclissi o, ancora, quanto tempo impiegherà un punto materiale a cadere da 10 metri di altezza nel vuoto in un qualsiasi punto della terra, note che siano latitudine ed altitudine. Ma davvero il potere predittivo della fisica si basa sulla misura di grandezze 
ottenute mediante procedimenti oggettivi di confronto con una unità di misura?

Facciamo un altro esempio per meglio chiarire il concetto: mentre è possibile calcolare con grande esattezza dove cadrà il punto materiale (possiamo pensare per semplicità ad una sfera di densità uniforme) dell'esempio precedente (anche tenendo conto dell'eventuale rotazione terrestre e quindi della deviazione verso est della caduta rispetto alla verticale) ed in quanto tempo, immaginiamo di fare il seguente esperimento. In una giornata ventosa saliamo su un grattacielo e lasciamo cadere una foglia dall'ultimo piano. Nessuna persona di buon senso è in grado di dirci dove cadrà la foglia e soprattutto in quanto tempo. Vuol forse dire che non stiamo misurando in modo oggettivo? Certamente no, il punto è che vi sono altre variabili in gioco, escluse nell'esperimento ideale descritto in precedenza, quali la forma della foglia, il suo attrito con l'aria e soprattutto la direzione e intensità del vento in ogni punto dello spazio. In che modo la fisica ci aiuta? Ovvero, come può rendere predicibile tale fenomeno naturale? In teoria è relativamente semplice: basta aggiungere in ogni punto dello spazio e ad ogni istante di tempo un campo di forze (ovvero un vettore) che ci fornisca in quel punto e in quell'istante l'intensità e soprattutto la direzione della forza. In pratica, non basterebbe il computer più potente del mondo per poter prevedere questo fenomeno, nemmeno con un grande errore sul possibile risultato. Mi si dirà che tutto ciò non appartiene ai compiti riconosciuti ai fisici, i quali dovrebbero semplicemente formulare le leggi fondamentali che governano i fenomeni naturali. Parafrasando quella che è un'idea abbastanza ricorrente tra la comunità dei fisici: compito dei fisici è scrivere le equazioni, non risolverle! Permettetemi di dissentire da questo tipo di atteggiamento: un fisico deve essere in grado, a mio parere, di fornire modelli matematici in grado di prevedere, con un errore stimabile, l'esito di certi fenomeni naturali. Se si assume questa, opinabile ovviamente, come definizione del ruolo del fisico (almeno di quello teorico) si comprende ad esempio come non si discosti molto da quello dell'economista che deve prevedere di quanto si discosterà, in percentuale, il Prodotto Interno Lordo dell'Italia nel prossimo anno rispetto a quello dell'anno corrente.

Il fisico e l'economista dunque cercano entrambi di fare delle previsioni basandosi su modelli matematici, in sostanza su equazioni tra variabili che possono essere "osservabili", o perlomeno riconducibili a grandezze osservabili, cioè misurabili. 
Ho qui introdotto un altro concetto che forse risulterà essere fondamentale per capire le eventuali differenze tra i rispettivi poteri predittivi: il concetto di osservabile fisica. Una osservabile in fisica è una grandezza che può essere misurata direttamente utilizzando il metodo scientifico descritto in precedenza e dovuto a Galileo. Questo ovviamente non toglie che alcune fondamentali equazioni della fisica non contengano come variabili delle osservabili: mi riferisco, nello specifico, all'equazione fondamentale della fisica microscopica moderna, nota come equazione di Schrodinger, che si riferisce ad una grandezza, funzione del punto spaziale $\mathrm{x}$ e del tempo $\mathrm{t}$, detta $\Psi(\mathrm{x}, \mathrm{t})$ o ampiezza di probabilità che non ha diretto significato fisico. La teoria ci dice però come relazionarsi agli esperimenti: il modulo elevato al quadrato della stessa rappresenta la densità di probabilità di trovare la particella microscopica nel punto dello spazio $\mathrm{x}$ all'istante di tempo t.

Un altro elemento di disturbo fa la sua comparsa: la probabilità. Come è possibile che una teoria fisica sia in grado di fornire, al meglio, solo la probabilità relativa ad una certa misura?

Ripeto meglio la cosa perché a mio avviso è essenziale: il risultato di una misura fatta nelle stesse identiche condizioni (stesso apparato, stesse condizioni iniziali) riesce a fornire solo la probabilità relativa al verificarsi o meno dell'evento.

Abbiamo forse a che fare anche qui, come nell'esempio precedente, con variabili "nascoste" come la velocità del vento, che ci impediscono di determinare esattamente il risultato dell'esperimento? La risposta è no: la Meccanica Quantistica è una teoria irriducibilmente statistica ed ogni teoria che preveda l'uso di variabili aggiuntive ma ignote che possa essere concepita è destinata all'insuccesso (è stato dimostrato che ciò sarebbe possibile solo rinunciando al principio di località).

Fatemi fare un passo indietro riguardo al concetto e all'uso della probabilità in fisica. Viene spesso scritto che la statistica, all'interno della matematica, rappresenta la teoria dell'ignoranza, ovvero che la si deve introdurre qualora il sistema sia così complesso da poter sopperire a questo deficit di informazioni solo con concetti di tipo statistico. Permettetemi ancora una volta di dissentire da questo punto di vista.

Per chiarire meglio questa posizione ricorriamo ancora una volta ad un esempio preso dalla fisica. Supponiamo di voler descrivere il comportamento di un certo volume di gas ad una data pressione e temperatura. La relazione che lega tra loro queste grandezze macroscopi- 
che (ovvero misurabili direttamente in laboratorio) pressione, temperatura e volume si chiama equazione di stato e la scienza che studia le varie trasformazioni tra queste (e altre ) grandezze macroscopiche si chiama Termodinamica. I successi ed i risultati ottenuti da questa disciplina sono sotto gli occhi di tutti nonostante questa equazione non ci dica nulla sulle leggi microscopiche che governano tali fenomeni. Progressi ancora più significativi si registrarono però quando fu possibile collegare i meccanismi di interazione tra i singoli costituenti microscopici (gli atomi o le molecole che compongono i gas) e tali leggi microscopiche. Storicamente ciò fu dovuto a Boltzmann che insieme a Gibbs contribuì in modo determinante alla nascita della cosiddetta Meccanica Statistica il cui scopo fu proprio quello di riuscire a creare un ponte tra le leggi microscopiche della Meccanica, a cui sono soggetti i singoli atomi, e le leggi della Termodinamica a cui sono soggette le variabili macroscopiche. Comprendere come i meccanismi microscopici influiscano sul comportamento macroscopico non costituisce un mero esercizio accademico: esso fornisce gli ingredienti indispensabili per la costruzione di quel modello matematico che può avere, o meno, potere predittivo.

E' altresì chiaro che l'uso della statistica in questo caso non è dovuto alla nostra ignoranza sullo stato iniziale (che pure esiste) quanto alla totale inutilità che una tale conoscenza avrebbe per prevedere il comportamento macroscopico. Anche se potessimo avere a disposizione un supercomputer in grado di calcolare tutte le traiettorie dei miliardi di miliardi di particelle racchiuse entro il volume dato, tale conoscenza risulterebbe irrilevante per poter prevedere, ad esempio, quale sia la temperatura del gas. La temperatura viene cioè ad assumere un duplice aspetto: da una parte quella di una grandezza macroscopica che si misura con un termometro, ma nel contempo, microscopicamente, assume il carattere di energia cinetica media delle molecole o degli atomi che costituiscono il gas. E con media si intende ovviamente il noto concetto statistico.

Anche qui è impensabile, ma soprattutto inutile, misurare l'energia cinetica di ogni singola molecola, mentre il valor medio su tutto l'insieme rappresenta una grandezza osservabile e misurabile sperimentalmente.

Nel caso della Meccanica Quantistica, o almeno di quella che è l'attuale interpretazione di essa (universalmente accettata ma non condivisa nel passato anche da fisici illustri quali Einstein) la situazione è 
ancora peggiore, nel senso che l'interpretazione statistica non è semplicemente più utile ma addirittura inevitabile!

Riassumiamo brevemente quanto detto fino ad ora in questa prima parte: il fisico si occupa della costruzione di opportuni modelli matematici, con la presunzione di poter essere in grado di fornire delle previsioni riguardo l'accadimento di certi fenomeni naturali. Inoltre utilizza, nella maggior parte dei casi, metodi investigativi di tipo statistico.

Fino ad ora abbiamo ignorato l'aspetto modellistico soprattutto in relazione con il grado di previsione raggiungibile. Diciamo, in modo piuttosto grossolano, che i modelli matematici studiati in fisica possono essere suddivisi in due classi distinte: deterministici e stocastici. Si potrebbe essere indotti, a questo punto, a mettere in relazione la predicibilità con l'utilizzo di un modello appartenente alla prima classe piuttosto che all'altra. In realtà, come spesso accade, le cose sono molto più complesse.

Vediamo in dettaglio le due tipologie suddette. I modelli cosiddetti stocastici, sono quelli in cui l'effetto delle forze in gioco viene introdotto nella forma di una variabile casuale dotata di una certa distribuzione di probabilità. In fisica, ancora una volta, forse il primo a farne uso esteso è stato Einstein, nella sua descrizione del moto Browniano. Particelle microscopiche (qualche micron) immerse in una sospensione liquida ad una certa temperatura sono soggette ad un moto irregolare, (una traiettoria a zig-zag) che rende assolutamente impossibile prevedere con esattezza la loro posizione futura. In realtà oggi sappiamo che il loro movimento è determinato dalle molte collisioni casuali che la particella subisce da parte delle molecole del liquido (o gas) in cui è immersa.

Supponendo che la forza impressa dalle molecole alla particella sia semplicemente casuale, un rumore bianco come viene detto in termini matematici, è possibile determinare in modo statistico la probabilità di trovare la particella ad un certo istante temporale entro una qualsiasi sfera di raggio $\mathrm{R}$ centrata sulla posizione iniziale (in realtà Einstein, studiando lo spostamento quadratico medio della particella riuscì a dare una caratterizzazione sperimentale esplicita del numero di Avogadro, ovvero del numero di particelle contenute in una mole di sostanza). L'equazione che si ottiene, detta equazione di Langevin, è alla base di importanti teorie fisiche, ad esempio la Reaction Rate Theory. Importanti settori della fisica utilizzano in modo estensivo concetti statistici, come ad esempio la teoria delle matrici a caso che ha fornito 
importanti risultati nel campo della fisica nucleare. Modelli matematici in cui una o più variabili sono inserite ad hoc, avendo certe distribuzioni di probabilità derivate da alcune semplici osservazioni o requisiti fisici minimali sono abbastanza comuni in fisica e solitamente forniscono indicazioni molto utili riguardo la possibilità di effettuare certe misure ed ottenere conseguentemente certi valori.

Questo è un punto delicato su cui vorrei fare alcune osservazioni. In fisica, anche se gli elementi casuali vengono introdotti in modo arbitrario nella teoria (o nell'insieme di equazioni che sono alla base del modello matematico costruito), occorre sempre relazionarli con opportune osservabili fisiche. Ad esempio, nel moto Browniano, la correlazione tra le posizioni della particella a due diversi istanti temporali si può mettere in relazione con la temperatura del fluido in cui è immersa.

In questo senso le assonanze con il mondo dell'economia sono numerose.

Ma c'è di più. Infatti, anche i cosiddetti modelli deterministici, se studiati a fondo ed in una certa ottica non lo sono poi così tanto! Ma come è possibile che un modello deterministico dia luogo a previsioni non deterministiche o casuali?

Facciamo un passo indietro e partiamo dalla stessa Meccanica Classica che tanti successi ha mietuto a partire dal secolo scorso.

Si è soliti citare in quest'ambito il motto di Laplace, il quale contemplando la bellezza delle equazioni di Newton, affermava che l'unico ruolo di Dio era quello di un grande orologiaio che mette in moto tutti i meccanismi dell'universo. Sotto questa sovraumana carica l'universo poteva evolvere in un modo assolutamente deterministico, soggetto alle sole leggi di interazione tra le sue particelle costituenti: fissate le condizioni iniziali (velocità e posizioni) di tutti i punti ad un qualsiasi istante di tempo (detto iniziale) si potrà prevedere dove saranno tutti i punti ad un qualsiasi istante finale (note che siano le leggi delle forze tra i vari elementi). Non c'è alcuna libertà in tale universo, tutto è deterministicamente prevedibile. La conclusione di Laplace segue dal fatto che le equazioni di Newton per un sistema di $\mathrm{N}$ particelle sono un sistema di $\mathrm{N}$ equazioni differenziali del secondo ordine (oppure, in modo del tutto analogo $2 \mathrm{~N}$ equazioni del primo ordine) e che quindi la soluzione esiste ed è unica assegnando le condizioni iniziali relative alle posizioni e alle velocità di ogni singolo punto ad un qualsiasi istante di tempo t (problema di Cauchy).

In realtà il primo ad infrangere il sogno di Laplace fu un altro 
grande fisico matematico del secolo scorso: Poincaré, con i suoi studi sul problema dei 3 corpi soggetti ad una reciproca interazione gravitazionale. Purtroppo Poincaré non aveva a sua disposizione, un computer (strumento indispensabile oggigiorno), così che i suoi studi rimasero a lungo quasi ignorati dalla comunità dei fisici. Oggi si sa, invece, che la caratteristica principale delle equazioni di Newton è costituita dalla loro non linearità la quale, a sua volta porta alla cosiddetta dipendenza sensibile dalle condizioni iniziali. Dal punto di vista matematico il problema è ben noto e consiste nell'affermare che le soluzioni future, pur essendo perfettamente deterministiche dipendono in modo esponenziale dall'errore sulle condizioni iniziali. La rilevanza sul piano fisico è enorme: siccome la misura di una qualsiasi osservabile fisica può essere effettuata solo a meno di un certo errore sperimentale, ne discende, inevitabilmente, che ogni previsione futura sarà affetta da una incertezza esponenzialmente grande nel tempo: tanto più saranno richieste previsioni a lungo termine tanto più l'esito sarà incerto. In altri termini: ogni sistema non lineare possiede una certa caratteristica, detto tempo di Lyapunov, al di sotto del quale sarà possibile fare previsioni e controllare l'errore su di esse, mentre, al di sopra di esso, dovremo abbandonare ogni tipo di certezza deterministica. Ogni sistema non lineare è caratterizzato da un proprio tempo di Lyapunov, che può essere piccolo: qualche secondo per un fluido turbolento o qualche giorno per le previsioni meteorologiche, o molto grande (qualche milione di anni) per i corpi siderali (e ciò spiega la grande precisione con cui riusciamo a prevedere il moto dei pianeti).

La gravità della situazione non si limita però alla sola dipendenza sensibile dal dato iniziale, ma anche a quello che si chiama effetto delle piccole perturbazioni (noto al grande pubblico come effetto butterfly). E' possibile che il battito delle ali di una farfalla a Pechino provochi un maremoto nel Golfo del Messico? Matematicamente il problema è molto complesso, una generica piccola perturbazione che facciamo agire sul nostro sistema (e tali perturbazioni esistono sempre: si ricordi che il modello è sempre un'astrazione della realtà che è ovviamente più complessa ed articolata) ha come effetto generico quello di produrre caos, ovvero indeterminismo sul comportamento futuro. E quando si è in presenza di indeterminismo l'unica cosa sensata che possiamo fare è ricorrere ad un approccio di tipo statistico, il quale ci permetterà di fornire previsioni esatte circa la probabilità con cui certi eventi si potranno verificare o meno. 
Volendo tirare delle conclusioni: la fisica si occupa della descrizione della realtà. Tale descrizione viene fatta attraverso modelli matematici che riescono ad estrapolare le leggi nascoste che governano i fenomeni naturali e a coglierne gli aspetti essenziali. I modelli possono essere deterministici o stocastici ma, spesso e volentieri, per entrambi si riesce al più ad avere risposte di tipo probabilistico. Un modello deterministico è sempre costruito a partire da grandezze osservabili, ovvero direttamente o indirettamente misurabili sperimentalmente. Un modello stocastico dipende invece da grandezze ignote di cui si ipotizza una certa distribuzione di probabilità la quale risulta però essere direttamente collegata alle osservabili.

E' adesso compito degli economisti capire se, e in che modo, la fisica può aiutare ad affrontare certe problematiche nel complesso campo economico. 